Nox $2^{-/-}$ageing mice. Compared with young WT mice, WT ageing mice had significantly high levels of fasting serum insulin and this was accompanied with delayed clearance of glucose $(\mathrm{P}<0.05)$ indicating insulin resistance. However, no indication of insulin resistance was found in Nox2 $2^{-/-}$ageing mice. The endothelial function was examined using aortic rings in an organ bath. Compared to the young controls, there was a significant decrease in the endothelium-dependent relaxation to acetylcholine in WT ageing aortas (Emax 72\% for young and 64\% for ageing, $\mathrm{P}<0.05$ ). However, endothelial function was well preserved in $\mathrm{Nox}^{-1-}$ ageing aortas (Emax 83\% for young and $80 \%$ for ageing. The ROS production was then measured in aortic sections by DHE fluorescence and a significant increase was found in ROS production in WT ageing aortas as compared to WT young controls $(\mathrm{P}<0.05)$. However, there was a significantly lower level of ROS production $(\mathrm{P}<0.05)$ in both age-matched $\mathrm{Nox}^{-/-}$aortas. In conclusion, Nox2-derived oxidative stress plays an important role in ageing-associated metabolic disorders and vascular dysfunction, and targeting Nox2 represents a valuable therapeutic strategy to treat these aging-related diseases.

\section{A CRUCIAL ROLE OF NOX2-DERIVED OXIDATIVE STRESS IN AGEING-ASSOCIATED METABOLIC DISORDERS AND VASCULAR DYSFUNCTION}

S Cahill-Smith, J M Li University of Surrey

doi:10.1136/heartjnl-2013-304019.169

Ageing has been recognised to be a major risk factor for the development of cardiovascular disease and growing evidence suggests a role for oxidative stress. NADPH oxidase 2 has been reported to be a major source of reactive oxygen species (ROS) generation in the cardiovascular system, however, the role of this enzyme in age-related metabolic disorders and vascular diseases remains unclear. In this study we used age-matched wild-type (WT) and Nox2-deficient (Nox2 ${ }^{-/}$) mice on a C57BL/6 background at young (3-4 month) and ageing (20-24 month) to investigate the role of Nox2 in age-related oxidative stress, metabolic disorders and vascular dysfunction. There was an age-related increase in blood pressure in WT mice (126 $\mathrm{mmHg}$ for young and $148 \mathrm{mmHg}$ for ageing) $(\mathrm{P}<0.05)$; however the blood pressure remained at lower levels in 